\title{
STATISTICAL ESTIMATION OF THE DYNAMICS OF WATERSHED DAMS
}

\author{
ZBISŁAW TABOR \\ Institute of Applied Computer Science \\ Cracow University of Technology, al. Jana Pawła II 37, 31-864 Cracow, Poland \\ e-mail:ztabor@pk.edu.pl
}

\begin{abstract}
In the present study the notion of watershed contour dynamics, defined within the framework of mathematical morphology, is examined. It is shown that the dynamics are a direct measure of the "sharpness" of transition between neighboring watershed basins. The expressions for the expected value and the statistical error of the estimation of contour dynamics are derived in the presence of noise, based on extreme value theory. The sensitivity of contour dynamics to noise is studied. A statistical approach to the notion of contour dynamics is developed and a definition of statistical dynamics is proposed.
\end{abstract}

Keywords: hierarchical segmentation, contour dynamics, mathematical morphology, statistical analysis.

\section{Introduction}

Segmentation and contour extraction are essential procedures for many image analysis tasks. The general segmentation problem involves assigning labels to the pixels of an analyzed image in such a way that two pixels of an image have the same label if and only if they belong to the same homogeneous regions of the scene. Obviously, the most ambiguous step of segmentation is deciding whether two pixels are or are not within a "homogeneous" region.

Many techniques have been proposed to deal with the image segmentation problem. According to the edgebased approach, object edges are detected first and then grouped into object boundaries. Edge-based local approaches, which use gradient information to obtain the edges, frequently suffer from either false acceptance of non-edge pixels as edge ones or classifying edge pixels as non-edge ones. These errors, which arise due to noise, generally do not allow generating closed curve representations of object boundaries.

The watershed transform (Beucher and Lantuéjoul, 1979) is a powerful edge-based method of segmentation, developed within the framework of mathematical morphology. It overcomes the problem of non-closed object boundaries. Watershed basins and watershed dams are obtained as the result of the watershed transform. However, watershed segmentation typically leads to strong oversegmentation of an analyzed image. Due to noise, there are significantly more watershed basins than objects in the scene and, consequently, some watershed dams do not correspond to real edges. Usually, however, it can be assumed that some watershed dams are already aligned with true edges and the remaining problem is to reject the false dams. Many methods have been proposed for that purpose, among other things, watersheds from markers (Vincent and Soile, 1991; Beucher and Meyer, 1993). In that case, the watershed transform starts from a pre-selected set of markers rather than from all regional minima. The selected markers are used to modify the original landscape by means of geodesic reconstruction so that the regional minima of the reconstructed image are located only at the imposed markers.

Other methods of dealing with oversegmentation include different hierarchical or hybrid approaches (Beucher, 1994; Najman and Schmitt, 1996; Haris et al. 1998). Generally, the aim of hierarchical segmentation based on the watershed transform is to group neighboring watershed basins through the use of some criteria, involving the properties of both these basins and the dam separating them. To assign importance to watershed dams, the notion of contour dynamics has been developed within the framework of mathematical morphology (Najman and Schmitt, 1996). However, as suggested by Haris et al. (2001), the morphological value of contour dynamics can be a poor estimate of real contour importance, especially in the case of noisy images, since it depends only on the difference of gray-level intensities in some two pixels. Except for this suggestion, there is, however, no systematic study of the sensitivity of contour dynamics with 
respect to noise.

This work presents an interpretation of the concept of contour dynamics as well as a statistical approach to the problem of estimating contour dynamics. Errors in the estimation of contour dynamics and their dependence on noise intensity are calculated based on extreme value theory (Burry, 1975).

\section{Background of watershed transform methods}

2.1. Watershed transform. Let $A$ be a set, and $a$ and $b$ two points of $A$. The geodesic distance $d_{A}(a, b)$ in $A$ is the lower bound of the length of the paths connecting $a$ and $b$ in $A$. In the case of a digital image, the distance is inherited from the underlying grid. The geodesic distance $d_{A}(a, B)$ from a point $a$ to the set $B$ is defined as

$$
d_{A}(a, B)=\min _{c \in B} d_{A}(a, c) .
$$

Let

$$
B=\bigcup_{i=1}^{k} B_{i} \subset A,
$$

where $B_{i}$ are connected components of $B$. The geodesic influence zone $i z_{A}\left(B_{i}\right)$ of $B_{i}$ is defined as

$$
\begin{aligned}
i z_{A}\left(B_{i}\right)=\{a \in A, \forall j & \in[1, k] \backslash\{i\}, \\
& \left.d_{A}\left(a, B_{i}\right)<d_{A}\left(a, B_{k}\right)\right\} .
\end{aligned}
$$

The union of the influence zones $i z_{A}\left(B_{i}\right)$ of $B_{i}$ is denoted by $I Z_{A}(B)$ (Fig. 1). Finally, let $f: A \rightarrow Z$ be a bounded function from $A$ to the set of natural numbers, $h_{\min }=$ $\min f, h_{\max }=\max f,[f]^{h}=\{a \in A, f(a) \leq h\}$. The set of catchment basins of $f$ is the set $X_{h_{\max }}$ obtained after the following recurrence:

$$
\begin{aligned}
X_{h_{\text {min }}} & =[f]^{h_{\text {min }}}, \\
X_{h+1} & =\text { Reg_Min } \\
h+1 & (f) \cup I Z_{[f]^{h}+1}\left(X_{h}\right),
\end{aligned}
$$

where Reg_Min ${ }_{h+1}(f)$ denotes the union of the regional minima of $f$ at the level $h+1$. A regional minimum $M$ at the intensity $h$ is a connected set of pixels with the intensity $h$, such that it is impossible to reach a pixel with an intensity $h^{\prime}<h$ starting from $M$ without having to pass a pixel with an intensity $h^{\prime \prime}>h$. The watershed of $f$ is the complementary of $X_{h_{\max }}$ (Fig. 2).

2.2. Hierarchical segmentation. An undesirable property of the watershed transform is that it usually leads to strong oversegmentation. According to the notion of hierarchical segmentation (Beucher, 1994), one computes the importance of the contours with respect to some criteria, instead of preventing oversegmentation. The importance of a contour is used to define a hierarchy of partitions. Formally, a hierarchy $P=\left\{P_{i}, i \in\{1,2, \ldots, k\}\right\}$
Fig. 1. Influence zone of every connected subset of an image (e.g., black disks) includes image pixels which are closer to a given subset than to the other one. Gray lines denote pixels which are equidistant relative to at least two connected subsets of an image. These lines form a skeleton by influence zones.
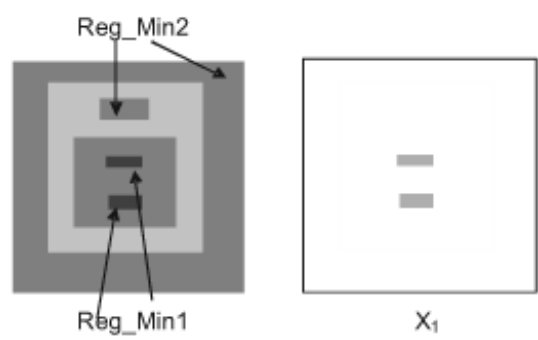

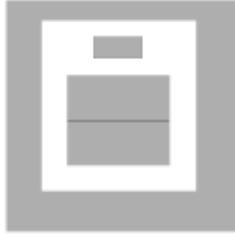

$\mathrm{X}_{2}$

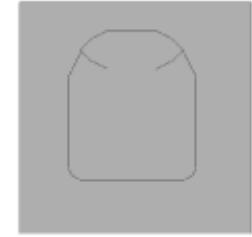

$\mathrm{X}_{3}$
Fig. 2. Successive steps of the watershed algorithm. Gray levels vary from 1 (darkest region) to 3 (brightest region). Regional minima are pointed with arrows. Black lines, consisting of pixels which are not within influence zones of any regional minima, constitute watershed dams.

on the set $A$ is a family of partitions

$$
\begin{aligned}
P_{i}=\left\{P_{i j}:\right. & \bigcup_{j} P_{i j}=A, \\
& \left.\forall m, n, m \neq n: P_{i m} \neq P_{i n}\right\}
\end{aligned}
$$

of $A$, such that $i>j$ implies that any region of $P_{i}$ is a disjoint union of regions of the partition $P_{j}$. While the hierarchy is defined, the features at a given hierarchy level can be extracted from the scene.

A first example of hierarchical segmentation is the waterfall algorithm (Beucher and Meyer, 1993; Beucher, 1994). According to that method, the subsequent levels of hierarchy are built based on markers constructed from watershed dams of the preceding hierar- 
chy level. Another hierarchy can be constructed relying on the notion of the dynamics of a regional minimum (Grimaud, 1992). The dynamics $d y n(M)$ of a regional minimum $M$ are equal to the highest $t$ such that $M$ is included in some regional minimum of the geodesic reconstruction by the erosion $E_{f}^{\infty}\left(f_{t}\right)$ of $f_{t}$ over $f$, where $f_{t}(a)=f(a)+t$. Consequently, one can create hierarchy assigning to every dam of a watershed the maximal value of $t$ for which the dam belongs to the watershed of $E_{f}^{\infty}\left(f_{t}\right)$ (Najman and Schmitt, 1996). More specifically, let $a$ be the lowest intensity point on a watershed dam separating two basins $Y_{i}$ and $Y_{j}$. A set $\operatorname{Bas}(a) \subset Y_{i} \cup Y_{j}$ can be constructed:

$$
\begin{aligned}
\operatorname{Bas}(a)=\{b: \exists \gamma & , \gamma(0)=a, \gamma(1)=b, \\
& \forall s \in(0,1]: f(\gamma(s))<f(a)\}
\end{aligned}
$$

The set $\operatorname{Bas}(a)$ can be divided into several (at least two) connected components $B_{i}$ :

$$
\operatorname{Bas}(a)=\bigcup_{i} B_{i}
$$

Then the morphological dynamics $\operatorname{dyn}(C)$ associated with the contour $C$, to which the saddle point a belongs, are given by the equation

$$
\operatorname{dyn}(C)=\min _{i} \max _{b_{i} \in B_{i}}\left\{f(a)-f\left(b_{i}\right)\right\} .
$$

Basically, morphological dynamics of a watershed dam are the depth (measured relative to the lowest point of the contour) of the shallowest basin neighboring to the dam (Fig. 3). Recently, also another definition of contour dynamics was proposed (Haris et al., 2001):

$$
D_{\text {mean }}(C)=\langle f(C)\rangle-\min _{k, b_{i} \in B_{k}} f\left(b_{i}\right),
$$

where $\langle f(C)\rangle$ is the mean value of gray-level intensities along the contour $C$. It was argued that $D_{\text {mean }}(C)$ should be possibly more robust with respect to noise, in comparison with $\operatorname{dyn}(C)$, because the latter depends only on graylevel intensities in some two pixels.

\section{Interpretation of the dynamics of water- shed contours}

Below, the robustness of the two definitions of contour dynamics with respect to noise is studied. It is assumed that the analyzed image $A$ (a subset of $Z^{2}$ ) contains only two regions of constant (on average) gray-level intensity, separated by a boundary line. Later this assumption is relaxed. The gray-level intensity $f(x, y)$ within $A$ is given after appropriate rescaling in the form

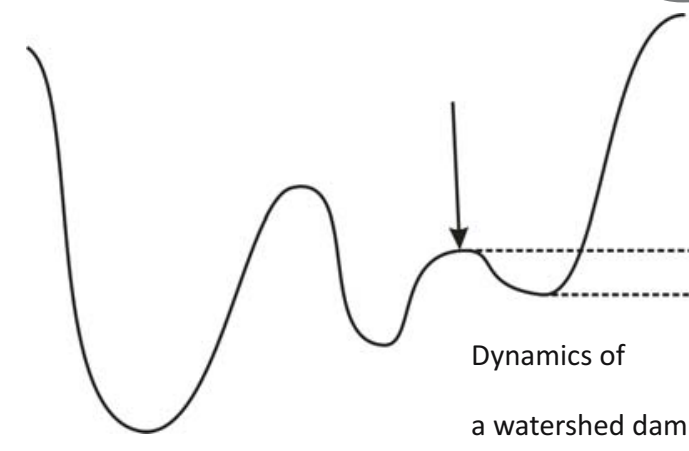

Fig. 3. Illustration of the notion of contour dynamics.

$$
\begin{aligned}
& f(x, y) \\
& =\eta+ \begin{cases}h_{\min }, & x<x_{\min }, \\
k\left(x-x_{\min }\right)+h_{\min }, & x_{\min } \leq x \leq x_{\max }, \\
k\left(x_{\max }-x_{\min }\right)+h_{\min }, & x>x_{\max },\end{cases}
\end{aligned}
$$

where $\eta$ is a zero mean noise term and the parameter $k$ measures the sharpness of transition between the two regions: a higher value of $k$ denotes a more sharp transition. Applying a derivative operator to $f(x, y)$ is a common way to obtain a magnitude of the gradient image, being the input for the watershed transform. In an ideal case of no noise, both definitions of contour dynamics, given in Eqns. (5) and (6), are equivalent and the value of contour dynamics is equal to the parameter $k$ in Eqn. (7), i.e., it is a direct measure of the sharpness of the boundary. Below, an expression for contour dynamics is derived under the assumption that $\eta$ is zero mean Gaussian noise with the standard deviation equal to $\sigma$.

Applying a central difference operator to the graylevel function defined in Eqn. (7), one obtains the magnitude of the gradient $E(x, y)$ in a pixel with the coordinates $(x, y)$ equal to

$$
\begin{aligned}
& E(x, y) \\
& = \begin{cases}\sqrt{\left(k+N_{1}\right)^{2}+N_{2}^{2}}, & x_{\min } \leq x \leq x_{\max }, \\
\sqrt{N_{1}^{2}+N_{2}^{2}}, & x<x_{\min } \text { or } x>x_{\max },\end{cases}
\end{aligned}
$$

where $N_{1}=0.5(\eta(x+1, y)-\eta(x-1, y)), N_{2}=$ $0.5(\eta(x, y+1)-\eta(x, y-1))$, and $\eta\left(x^{\prime}, y^{\prime}\right)$ is the value of the noise term in a pixel with the coordinates $\left(x^{\prime}, y^{\prime}\right)$. The probability density function (pdf) $p_{N_{i}}$ of the noise term $N_{i}(i=1,2)$ can be calculated in a usual way, given the pdf $p_{\eta}$ of $\eta$ :

$$
p_{N_{i}}(s)=\int p_{\eta}\left(t_{1}\right) p_{\eta}\left(t_{2}\right) P\left(s \mid t_{1}, t_{2}\right) \mathrm{d} t_{1} \mathrm{~d} t_{2},
$$


where $P\left(s \mid t_{1}, t_{2}\right)$ is the conditional probability that $s$ is equal to $0.5\left(t_{1}-t_{2}\right)$, for given values of $t_{1}$ and $t_{2}$. This term is trivially proportional to the Dirac delta function $\delta\left(s-0.5\left(t_{1}+t_{2}\right)\right)$. If $\eta$ is a zero mean Gaussian random variable with the standard deviation $\sigma$, then $N_{i}$ is also a Gaussian random variable with the standard deviation $\sigma / \sqrt{2}$. To calculate the pdf of $E(x, y)$, a random variable $s(s=E(x, y))$ is defined as $s=\sqrt{y_{1}^{2}+y_{2}^{2}}$, where $y_{1}=k+N_{1}, y_{2}=N_{2}$. The pdfs $p_{Y_{1}}$ and $p_{Y_{2}}$ of $y_{1}$ and $y_{2}$ are equal to

$$
\begin{aligned}
& p_{Y_{1}}\left(y_{1}\right)=\frac{1}{\sigma \sqrt{\pi}} \exp \left(-\frac{\left(y_{1}-k\right)^{2}}{\sigma^{2}}\right), \\
& p_{Y_{2}}\left(y_{2}\right)=\frac{1}{\sigma \sqrt{\pi}} \exp \left(-\frac{y_{2}^{2}}{\sigma^{2}}\right) .
\end{aligned}
$$

The pdf $p_{k}(s)$ of $s$ can be calculated from the equation

$$
p_{k}(s)=\int p_{Y_{1}}\left(y_{1}\right) p_{Y_{2}}\left(y_{2}\right) \delta\left(s^{2}-\left(y_{1}^{2}+y_{2}^{2}\right)\right) \mathrm{d} y_{1} \mathrm{~d} y_{2} .
$$

It can be shown, after switching to polar coordinates, that the distribution $p_{k}(s)$ of the gradient magnitudes $s$ in the range $x_{\min }<x<x_{\max }$ is given by

$$
\begin{aligned}
p_{k}(s)= & \frac{s}{\pi \sigma^{2}} \int_{-\pi}^{\pi} \exp \left(-\frac{(s-k \cos \theta)^{2}}{\sigma^{2}}\right) \\
& \cdot \exp \left(-\frac{k^{2} \sin ^{2} \theta}{\sigma^{2}}\right) \mathrm{d} \theta
\end{aligned}
$$

If the ratio $k / \sigma$ is sufficiently large, the damping factor $\exp \left(-k^{2} \sin ^{2} \theta / \sigma^{2}\right)$ is very close to zero except for $\theta \approx 0$. Then, replacing it that limit $\sin (\theta)$ by $\theta$, the damping factor can be integrated and the following approximation to $p_{k}$ can be obtained:

$$
p_{k}(s)=\frac{1}{\sqrt{\pi}} \frac{s}{k \sigma} \exp \left(-\frac{(s-k)^{2}}{\sigma^{2}}\right) .
$$

For $k / \sigma \rightarrow \infty$, one has $\int_{0}^{\infty} p_{k}(s) \mathrm{d} s=1$. In that limit, the mean value $\left\langle p_{k}\right\rangle$ and the standard deviation $\sigma_{p_{k}}$ of $s$ can be calculated from Eqn. (13). It follows that both these quantities are increasing functions of $\sigma$ (Fig. 4):

$$
\begin{aligned}
\left\langle p_{k}\right\rangle & =k+\frac{\sigma^{2}}{2 k}, \\
\sigma_{p_{k}} & =\sqrt{\frac{\sigma^{2}}{2}\left(1-\frac{\sigma^{2}}{k^{2}}\right)} .
\end{aligned}
$$

The distribution $p_{k}(s)$ is parameterized by the value of $k$ in Eqn. (7). In the limit $k=0$, an exact expression for the distribution $p_{\mathrm{bgr}}(s)$ of the gradient magnitudes $s$ in the range $x<x_{\min }$ or $x>x_{\max }$ is approached:

$$
p_{\text {bgr }}(s)=\frac{2 s}{\sigma^{2}} \exp \left(-\frac{s^{2}}{\sigma^{2}}\right)
$$

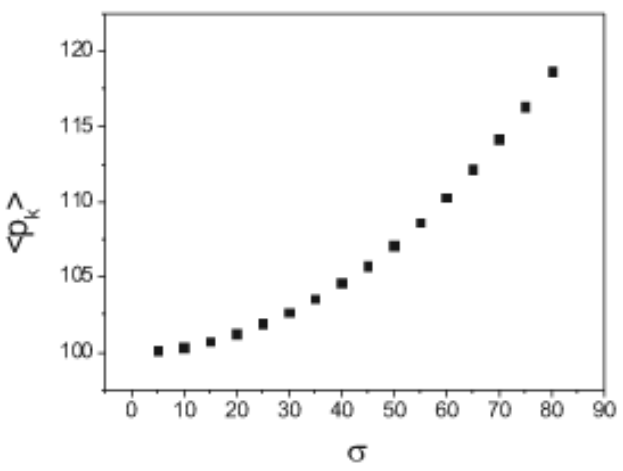

(a)

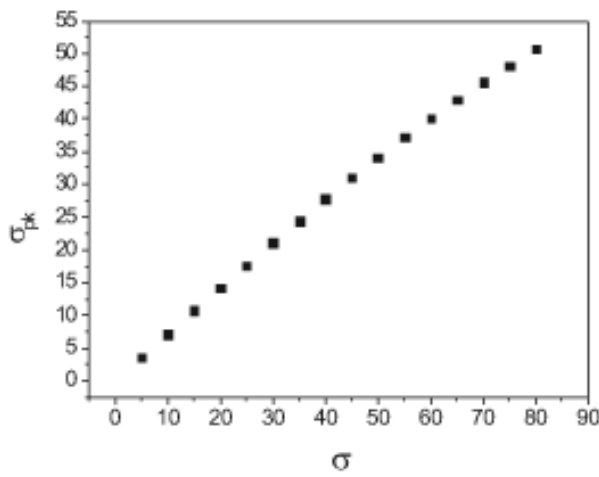

(b)

Fig. 4. Mean value (a) and standard deviation (b) of the distribution of gray-level intensities along an edge for the parameter $k$ in Eqn. (7) equal to 100 and Gaussian noise characterized by the standard deviation equal to $\sigma$.

with the mean value $\left\langle p_{\mathrm{bgr}}\right\rangle$ and the standard deviation $\sigma_{p_{\text {bgr }}}$ depending only on $\sigma$ :

$$
\begin{aligned}
\left\langle p_{\text {bgr }}\right\rangle & =\frac{\sigma}{2} \sqrt{\pi} \\
\sigma_{p_{\text {bgr }}} & =\frac{\sigma}{2} \sqrt{4-\pi} .
\end{aligned}
$$

It is reasonable to assume that the watershed transform, when applied to a gradient magnitude image of the gray-level function specified in Eqn. (7), produces watershed dams in edge regions, while watershed basins arise in regions of $f$ fluctuating around constant values. Then the distribution of gray-levels within dams and basins follows from Eqns. (13) and 15, respectively. Having these distributions determined, morphological dynamics of a dam can be estimated. For that purpose, it is assumed that a dam consists of $L_{\text {edge }}$ pixels while a watershed basin neighboring to the dam contains $L_{\mathrm{bgr}}$ pixels. Then the 
expected value of the dynamics of a dam $C$ is equal to

$$
\begin{aligned}
\langle\operatorname{dyn}(C)\rangle= & \left\langle\min \left(L_{\text {edge }}, p_{k}\right)\right\rangle \\
& -\left\langle\min \left(L_{\text {bgr }}, p_{\text {bgr }}\right)\right\rangle, \\
\left\langle D_{\text {mean }}(C)\right\rangle= & \left\langle p_{k}\right\rangle-\left\langle\min \left(L_{\mathrm{bgr}}, p_{\text {bgr }}\right)\right\rangle,
\end{aligned}
$$

where $\min \left(L_{\text {edge }}, p_{k}\right)$ denotes a minimal value from a series of $L_{\text {edge }}$ numbers sampled from the $p_{k}$ distribution and $\min \left(L_{\mathrm{bgr}}, p_{\mathrm{bgr}}\right)$ is a minimal value from a series of $L_{\mathrm{bgr}}$ numbers sampled from the $p_{\mathrm{bgr}}$ distribution. To calculate $\left\langle\min \left(L_{\mathrm{bgr}}, p_{\mathrm{bgr}}\right)\right\rangle$, one should notice that the probability $P\left(\min \left(L_{\mathrm{bgr}}, p_{\mathrm{bgr}}\right)<x\right)$ that $\min \left(L_{\mathrm{bgr}}, p_{\mathrm{bgr}}\right)$ is less than $x$ is equal to

$$
P\left(\min \left(L_{\mathrm{bgr}}, p_{\mathrm{bgr}}\right)<x\right)=1-\left(1-F_{\mathrm{bgr}}(x)\right)^{L_{\mathrm{bgr}}},
$$

where $F_{\mathrm{bgr}}(x)$, the cumulative distribution function of $p_{\text {bgr }}$, is equal to

$$
F_{\text {bgr }}(x)=1-\exp \left(-\frac{x^{2}}{\sigma^{2}}\right) .
$$

Given the cumulative distribution function of $\min \left(L_{\text {bgr }}, p_{\text {bgr }}\right) \quad$ (Eqn. (18), the expected value $\left\langle\min \left(L_{\mathrm{bgr}}, p_{\mathrm{bgr}}\right)\right\rangle$ and the standard deviation $\sigma_{\min \left(L_{\mathrm{bgr}}, p_{\mathrm{bgr}}\right)}$ of $\min \left(L_{\mathrm{bgr}}, p_{\mathrm{bgr}}\right)$ can be calculated:

$$
\begin{aligned}
\left\langle\min \left(L_{\mathrm{bgr}}, p_{\mathrm{bgr}}\right)\right\rangle & =\left\langle p_{\mathrm{bgr}}\right\rangle \frac{1}{\sqrt{L_{\mathrm{bgr}}}}=\frac{\sigma}{2} \sqrt{\frac{\pi}{L_{\mathrm{bgr}}}}, \\
\sigma_{\min \left(L_{\mathrm{bgr}}, p_{\mathrm{bgr}}\right)} & =\sigma_{p_{\mathrm{bgr}}} \frac{1}{\sqrt{L_{\mathrm{bgr}}}}=\frac{\sigma}{2} \sqrt{\frac{4-\pi}{L_{\mathrm{bgr}}}},
\end{aligned}
$$

where $\left\langle p_{\mathrm{bgr}}\right\rangle$ and $\sigma_{p_{\mathrm{bgr}}}$ are the expected value and the standard deviation of the $p_{\mathrm{bgr}}$ distribution. Assuming that $p_{k}$ is approximately a Gaussian with the mean and the standard deviation given in Eqn. (14), the distribution of $\min \left(L_{\text {edge }}, p_{k}\right)$ converges to the Gumbel distribution (Burry, 1975). Then it follows that the expected value $\left\langle\min \left(L_{\text {edge }}, p_{k}\right)\right\rangle$ and the standard deviation $\sigma_{\min \left(L_{\text {edge }}, p_{k}\right)}$ of $\min \left(L_{\text {edge }}, p_{k}\right)$ are equal to

$$
\begin{aligned}
\left\langle\min \left(L_{\text {edge }}, p_{k}\right)\right\rangle & =\left\langle p_{k}\right\rangle+\left(a_{L_{\text {edge }}} \gamma+b_{L_{\text {edge }}}\right) \sigma_{k}, \\
\sigma_{\min \left(L_{\text {edge }}, p_{k}\right)} & =-\sigma_{p_{k}} a_{L_{\text {edge }}} \frac{\pi}{\sqrt{6}},
\end{aligned}
$$

where $\left\langle p_{k}\right\rangle$ and $\sigma_{p_{k}}$ are the mean and the standard deviation of the $p_{k}$ distribution, respectively, $\gamma \approx 0.5772$ is the Euler constant, $a_{L_{\text {edge }}}$ and $b_{L_{\text {edge }}}$ are equal to

$$
\begin{aligned}
& b_{L_{\text {edge }}}=F^{-1}\left(\frac{1}{L_{\text {edge }}}\right) \\
& a_{L_{\text {edge }}}=F^{-1}\left(\frac{1}{a L_{\text {edge }}}\right)-b_{L_{\text {edge }}}
\end{aligned}
$$

$F^{-1}(\cdot)$ is the inverse of the cumulative distribution function of the standardized Gaussian distribution and $e=\exp (1) . \quad$ The values of $\left\langle\min \left(L_{\mathrm{bgr}}, p_{\mathrm{bgr}}\right)\right\rangle$ and $\left\langle\min \left(L_{\text {edge }}, p_{k}\right)\right\rangle$ are plotted vs. $\sigma$ for a fixed value of the number of pixels in Fig. 5 and vs. the number of pixels within an edge/basin for a fixed value of $\sigma=30$ in Fig. 6 . Combining Eqns. (14), (16), (17), (20) and (21), one has expressions for the dynamics in the limit of large $k / \sigma$ :

$$
\begin{aligned}
\langle\operatorname{dyn}(C)\rangle= & k+\sigma\left(\frac{\sigma}{2 k}+\frac{\left(\gamma a_{L_{\text {edge }}}+b_{L_{\text {edge }}}\right)}{\sqrt{2}}\right. \\
& \left.-\sqrt{\frac{\pi}{4 L_{\mathrm{bgr}}}}\right), \\
\left\langle D_{\text {mean }}(C)\right\rangle= & k+\sigma\left(\frac{\sigma}{2 k}-\sqrt{\frac{\pi}{4 L_{\mathrm{bgr}}}}\right),
\end{aligned}
$$

where terms proportional to $(\sigma / k)^{2}$ are neglected in the parentheses.

It follows from Eqn. (22) that in the regime of $p_{k}$ being approximately Gaussian, $D_{\text {mean }}(C)$ differs from $\operatorname{dyn}(C)$ only by the factor proportional to $\left(a_{L_{\text {edge }}} \gamma+\right.$ $\left.b_{L_{\text {edge }}}\right) \sigma$. Keeping in mind that for any given watershed dam $\left\langle\min \left(L_{\mathrm{bgr}}, p_{\mathrm{bgr}}\right)\right\rangle$ and $\left\langle\min \left(L_{\text {edge }}, p_{k}\right)\right\rangle$ are estimated from a measurement performed in a single pixel, while $\left\langle p_{k}\right\rangle$ is estimated from $L_{\text {edge }}$ measurements, the errors of estimating $\operatorname{dyn}(C)$ and $D_{\text {mean }}(C)$ are

$$
\begin{aligned}
\operatorname{Err}(\operatorname{dyn}(C)) & =\sqrt{\sigma_{\min \left(L_{\mathrm{edge}}, p_{k}\right)}^{2}+\sigma_{\min \left(L_{\mathrm{bgr}}, p_{\mathrm{bgr}}\right)}^{2}}, \\
\operatorname{Err}\left(D_{\text {mean }}(C)\right) & =\sqrt{\frac{\sigma_{p_{k}}^{2}}{L_{\text {edge }}}+\sigma_{\min \left(L_{\mathrm{bgr}}, p_{\mathrm{bgr}}\right)}^{2}} .
\end{aligned}
$$

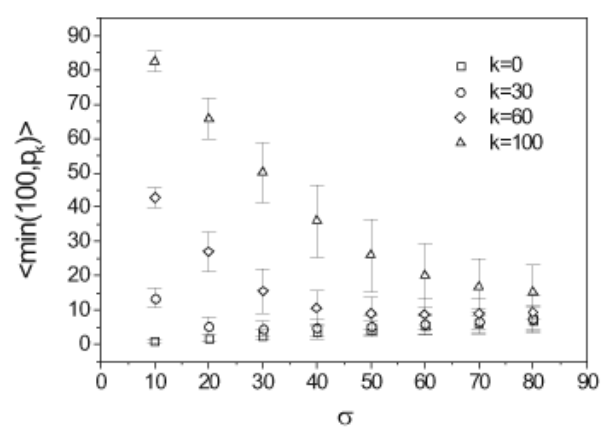

Fig. 5. Expected value of the minimum from 100 numbers sampled from the $p_{k}$ distribution, plotted vs. the standard deviation $\sigma$ of Gaussian noise. The case $k=0$ corresponds to the distribution $p_{\text {bgr }}$ (Eqn. (15)).

It follows from Eqn. (22) that the error of the mean of $L_{\mathrm{bgr}}$ numbers sampled from the distribution $p_{\mathrm{bgr}}$ is equal to the error of a single measurement of minimum from these $L_{\mathrm{bgr}}$ numbers, both given by the second equation of (20). Consequently, it is not statistically justified to replace $\min \left(L_{\mathrm{bgr}}, p_{\mathrm{bgr}}\right)$ in the definition of dynamics by some form of averaging over gradient magnitude values. On the other hand, it may be 


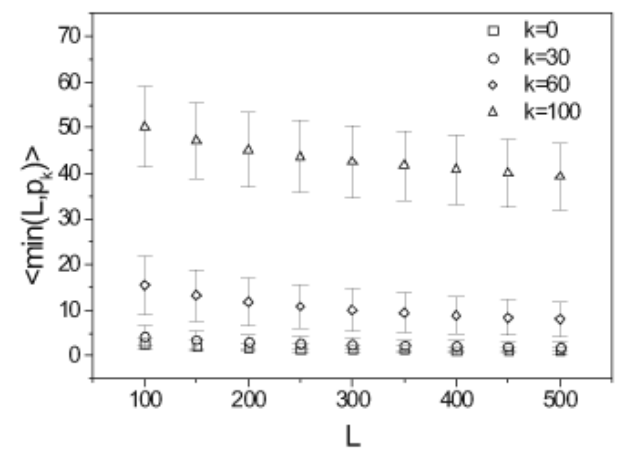

Fig. 6. Expected value of the minimum from $L$ numbers sampled from the $p_{k}$ distribution, plotted vs. $L$. The standard deviation $\sigma$ of Gaussian noise was equal to 30 .

advantageous to replace $\min \left(L_{\text {edge }}, p_{k}\right)$ by $\left\langle p_{k}\right\rangle$. To see this, it is shown below how the error of $\min \left(L_{\text {edge }}, p_{k}\right)$, equal to $\sigma_{\min }\left(L_{\text {edge }}, p_{k}\right)$, decreases with increasing $L_{\text {edge }}$. $\sigma_{\min \left(L_{\text {edge }}, p_{k}\right)}$ depends on $L_{\text {edge }}$ only through the factor $a_{L_{\text {edge }}}$, defined in Eqn. (22), which is a decreasing function of $L_{\text {edge. }}$ The cumulative distribution function $F$ of the standardized Gaussian distribution is related to the error function through the expression

$$
F(x)=\frac{1}{2}\left(1+\operatorname{erf}\left(\frac{x}{\sqrt{2}}\right)\right) .
$$

To calculate $b_{L_{\text {edge }}}$ and $a_{L_{\text {edge }}}$, an approximation to $F(x)$, correct for large $x$, can be used:

$$
(\operatorname{erf}(x))^{2} \approx 1-\exp \left(-x^{2}\right)
$$

Thus, from Eqns. (25) and (26), one has

$$
\begin{aligned}
& F\left(x_{1}\right)=\frac{1}{L_{\text {edge }}}=\frac{1}{2}\left(1+\sqrt{1-\exp \left(-\frac{x_{1}^{2}}{2}\right)}\right), \\
& F\left(x_{2}\right)=\frac{1}{e L_{\text {edge }}}=\frac{1}{2}\left(1+\sqrt{1-\exp \left(-\frac{x_{2}^{2}}{2}\right)}\right) .
\end{aligned}
$$

$a_{L_{\text {edge }}}$ is equal to the difference $x_{2}-x_{1}$ and it can be shown that $a_{L_{\text {edge }}}$ approaches for large $L_{\text {edge }}$,

$$
a_{L_{\text {edge }}} \approx\left(2 \cdot \ln \left(L_{\text {edge }}\right)\right)^{-0.5} .
$$

Consequently, because the error of $\min \left(L_{\text {edge }}, p_{k}\right)$ decreases like $\ln \left(L_{\text {edge }}\right)^{-0.5}$, while the error of $\left\langle p_{k}\right\rangle$ decreases like $\left(L_{\text {edge }}\right)^{-0.5}, D_{\text {mean }}(C)$ can be possibly more robust to noise than $\operatorname{dyn}(C)$.

There can be, however, a serious disadvantage of using either $d y n(C)$ or $D_{\text {mean }}(C)$ to assess contour dynamics. Namely, it follows from Eqn. (23) that for sufficiently large $L_{\mathrm{bgr}}, D_{\text {mean }}(C)$ increases with $\sigma$, which is undesirable because noise usually destroys edges but not amplifies them. $\langle\operatorname{dyn}(C)\rangle$ decreases with $\sigma$ as long as the absolute value of the negative term $\gamma a_{L_{\text {edge }}}+b_{L_{\text {edge }}}$ and thus $L_{\text {edge }}$ are sufficiently large. In fact, it is easy to see that it happens for all $\sigma, k$ and $L_{\text {edge }}$ for which $\sigma / k$ is small. The values of $\operatorname{dyn}(C)$ and $D_{\text {mean }}(C)$ are plotted vs. $\sigma$ in Fig. 7 together with the respective error bars.

Unfortunately, if $f(x, y)$ is piecewise linear, then both $\operatorname{dyn}(C)$ and $D_{\text {mean }}(C)$ may behave poorly under some circumstances. Indeed, if $f(x, y)$ is piecewise linear, then the distribution $p_{\text {mix }}(s)$ of the gradient magnitudes $s$ within an image is a mixture of $p_{k}$ distributions:

$$
p_{\text {mix }}(s)=\sum_{k=k_{\min }}^{k_{\max }} \pi_{k} \cdot p_{k}(s),
$$

with some minimal $k_{\min }$ and maximal $k_{\max }$ values of $k$. Then, if an image contains an edge with $k=k_{\max }=250$, separating regions of more slowly varying gray-levels $\left(k=k_{\min }=150\right)$, then both $\operatorname{dyn}(C)$ and $D_{\text {mean }}(C)$ may increase with $\sigma$ (Fig. 8).

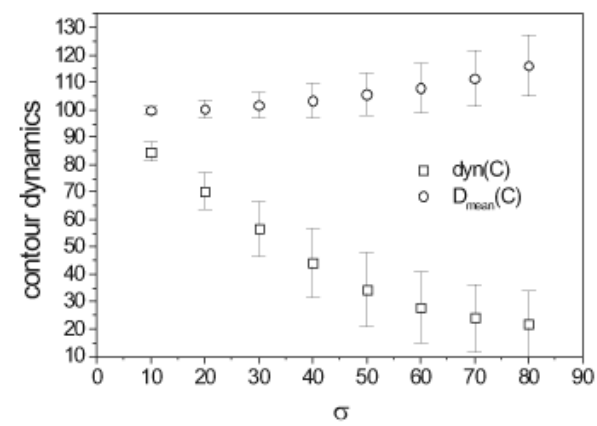

Fig. 7. Morphological (squares) and statistical (circles) contour dynamics plotted vs. the standard deviation $\sigma$ of Gaussian noise. The dynamics were calculated for gray-level intensity function given in Eqn. (7). In the calculations, the following values of parameters were used: $k=100$, $L_{\text {edge }}=40, L_{\text {bgr }}=1600$.

Another disadvantage of calculating dynamics, based on either Eqn. (5) or (6), is that dynamics so defined depend on the shape of the basin. Assuming again that $f(x, y)$ is piecewise linear and the distribution of gradient magnitudes is given by Eqn. (29), the minimum intensity $\min \left(L_{\mathrm{bgr}}, p_{\text {mix }}\right)$ within a basin consisting of $L_{\mathrm{bgr}}$ pixels is equal to

$$
\begin{aligned}
& \min \left(L_{\mathrm{bgr}}, p_{\operatorname{mix}}\right) \\
& =\min \left\{\min \left(L_{\mathrm{bgr}} \cdot \pi_{k}, p_{k}\right):\right. \\
& \left.\quad k \in\left\{k_{\min }, k_{\min }+1, \ldots, k_{\max }\right\}\right\} .
\end{aligned}
$$

Clearly, $\min \left(L_{\mathrm{bgr}}, p_{\text {mix }}\right)$ is in general dependent on all $\pi_{k} \mathrm{~s}$ and it follows from Eqns. (20) and (21) that, for ex- 


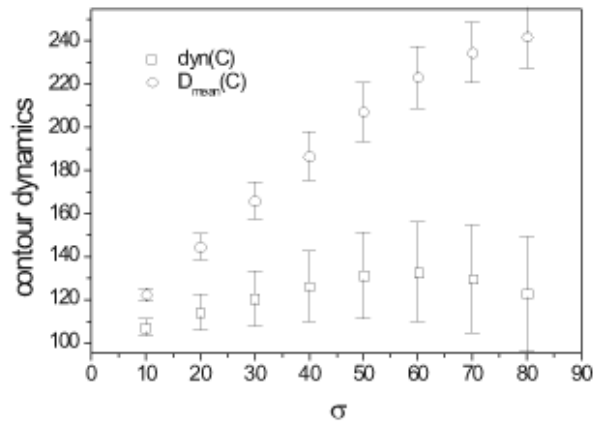

Fig. 8. Morphological (squares) and statistical (circles) contour dynamics plotted vs. the standard deviation $\sigma$ of Gaussian noise. The dynamics were calculated for a piecewise linear gray-level intensity function with $k_{\min }=$ $150, k_{\max }=250, L_{\text {edge }}=40, L_{\mathrm{bgr}}=1600$.

ample, its value for large $\pi_{k_{\min }}$ is different from the value for small $\pi_{k_{\min }}$.

Summarizing the results of the current section, it has been shown that in an ideal case the dynamics of a contour measure the difference $\Delta k$ of gradient magnitudes within a watershed dam and a watershed basin. If noise is present in an analyzed image, the dynamics defined in Enqs. (5) and (6) give, however, a biased estimate of that difference, systematically dependent on $\sigma$ as demonstrated in Eqn. (23). The dynamics depends also on the shape of the basin. Finally, the error of measuring $\operatorname{dyn}(C)$ decreases only logarithmically with the length of a watershed dam, while a smaller error of dynamics estimation is possible when averaging is performed instead of taking minimal values from gradient magnitudes.

\section{Vector-based definition of statistical dy- namics}

Even if the difference between gradient magnitudes within a basin and a watershed dam is zero, in the presence of noise contrast dynamics are nonzero, as demonstrated in the previous section. To obtain an unbiased, with this respect, estimate of contour dynamics, a two-dimensional random variable $\mathbf{Y}=\left(y_{1}, y_{2}\right)$ is introduced, such that the pdfs of the components $y_{1}$ and $y_{2}$ are generalizations of Eqn. (10) and thus the pdf of $\mathbf{Y}$ has the form

$$
p(\mathbf{Y})=\frac{1}{\sigma \sqrt{\pi}} \exp \left(-\frac{|\mathbf{Y}-\mathbf{k}|^{2}}{\sigma^{2}}\right),
$$

where $|\cdot|$ denotes a vector norm and the vector $\mathbf{k}$ can generally be a function of the coordinates $(x, y)$. Because the pdf of a sum of Gaussian distributions is also a Gaussian, averaging Ys over a watershed dam is a means of estimating the mean gradient vector $\mathbf{k}$. Hence, the magnitude $\left|\overline{\mathbf{Y}}_{\mathbf{D}}\right|$ of the arithmetic mean $\overline{\mathbf{Y}}_{\mathbf{D}}$, taken over Ys within a watershed dam (in contrast to the arithmetic mean of magnitudes, used in the definition of dynamics), provides an unbiased and independent on $\sigma$ estimate of the mean gradient magnitude along the dam. The standard deviation of $\left|\overline{\mathbf{Y}}_{\mathbf{D}}\right|$ is equal to $\sigma / \sqrt{L_{\text {edge }}}$.

Assuming an arbitrary shape of the watershed basin, the distribution of $Y_{s}$ within a basin is a mixture of Gaussians. To find that component of the mixture which corresponds to the minimal gradient magnitude within the basin, a growth process is performed as described in the next section. As the result of the process, a singleconnected set of pixels $S$ is created, which contains the pixel with the lowest gradient magnitude within the watershed basin and such that the gradient magnitudes within $S$ can be approximated by a plane of a constant gray level. Then the mean value $\left|\overline{\mathbf{Y}}_{\mathbf{S}}\right|$ of $\mathbf{Y}$ s within $S$ is an estimate of the mean gradient vector $\mathrm{g}$ within $S$. Finally, the magnitude $\left|\overline{\mathbf{Y}}_{\mathbf{S}}\right|$ is compared with $\left|\overline{\mathbf{Y}}_{\mathbf{D}}\right|$, as described below.

The key observation is that statistical dynamics can be assigned to a watershed dam by means of the following statistical testing procedure. Namely, it is shown below that the pdf of the variable $z$ defined by

$$
z=\frac{\left|\overline{\mathbf{Y}}_{\mathbf{D}}\right|-\left|\overline{\mathbf{Y}}_{\mathbf{S}}\right|}{\frac{\sigma}{\sqrt{2}} \sqrt{\frac{L_{\text {edge }}+L_{S}}{L_{\text {edge }} L_{S}}}}
$$

(where $L_{S}$ is the number of pixels within $S$ ) can be approximated by the standard normal distribution if $|k|=$ $|g|$. Thus, according to the null hypothesis, it is assumed that $|k|=|g|$ (a watershed dam is not related to a true edge). Then the value of $z$ is calculated and it is checked, based on the known pdf of $z$, whether the null hypothesis can be rejected given some significance level. Clearly, any given significance level corresponds to a level of the hierarchy of partitions: the level of the hierarchy is constructed by leaving only those watershed dams which are statistically significant.

Now, to show that the pdf of $z$ is a standard normal distribution, let us assume that the components of the mean gradient vector $\mathbf{k}$ within a watershed dam consisting of $L_{\text {edge }}$ pixels are equal to $k_{1}$ and $k_{2}$ in some orthogonal frame of reference. Then it follows that the pdf $p_{D}(s)$ of the magnitude $s$ of $\left|\overline{\mathbf{Y}}_{\mathbf{D}}\right|$ is equal to

$$
\begin{aligned}
p_{D}(s)= & \frac{L_{\text {edge }}}{\pi \sigma^{2}} \int_{0}^{\infty} r \mathrm{~d} r \int_{0}^{2 \pi} \mathrm{d} \theta \\
& \cdot e^{-\frac{L_{\text {edge }}}{\sigma^{2}}\left(\left(r \cos \theta-k_{1}\right)^{2}+\left(r \sin \theta-k_{2}\right)^{2}\right)} \\
& \cdot \delta(r-s) .
\end{aligned}
$$

Because the integral over the angle variable is invariant 
under rotations, $p_{D}(s)$ can be rewritten in the form

$$
\begin{aligned}
p_{D}(s)= & \frac{s \cdot L_{\text {edge }}}{\pi \sigma^{2}} \int_{0}^{2 \pi} \mathrm{d} \theta \\
& \cdot \exp \left(-\frac{L_{\text {edge }}}{\sigma^{2}}\left((s \cos \theta-|k|)^{2}\right.\right. \\
& \left.\left.+(s \sin \theta)^{2}\right)\right)
\end{aligned}
$$

which is analogous to Eqn. (12). The pdf $p_{S}(s)$ of the magnitude $s$ of $\overline{\mathbf{Y}}_{\mathbf{S}}$ can be found in a similar manner. Then a random variable $u$ is introduced such that $u=$ $\left|\overline{\mathbf{Y}}_{\mathbf{D}}\right|-\left|\overline{\mathbf{Y}}_{\mathbf{S}}\right|$. The pdf $p_{u}(u)$ of $u$ is equal to

$$
\begin{aligned}
p_{u}(u)= & \frac{L_{\mathrm{edge}} \cdot L_{S}}{\pi^{2} \sigma^{4}} \int_{0}^{\infty} \mathrm{d} s \cdot s(s-u) \\
& \cdot F\left(s-u,|k|, \frac{\sigma}{\sqrt{L_{\text {edge }}}}\right) F\left(s,|g|, \frac{\sigma}{\sqrt{L_{S}}}\right),
\end{aligned}
$$

where

$$
\begin{aligned}
& F(x, k, \sigma) \\
& \quad=\int_{0}^{2 \pi} \mathrm{d} \theta \cdot \exp \left(-\frac{(x \cos \theta-k)^{2}+x^{2} \sin ^{2} \theta}{\sigma^{2}}\right) .
\end{aligned}
$$

Assuming again that $k / \sigma$ is large and $|k|=|g|$, Eqn. (35) can be rewritten in the form

$$
\begin{aligned}
p_{u}(u)= & \frac{\sqrt{L_{\text {edge }} \cdot L_{S}}}{\pi \sigma^{2}|k|^{2}} \int_{0}^{\infty} \mathrm{d} s \cdot s(s-u) \\
& \cdot \exp \left(-\frac{L_{\text {edge }}(s-|k|)^{2}}{\sigma^{2}}\right) \\
& \cdot \exp \left(-\frac{L_{S}(s-u-|k|)^{2}}{\sigma^{2}}\right) .
\end{aligned}
$$

Changing the variable $s$ to $x=s-|k|$, one obtains

$$
\begin{aligned}
& p_{u}(u) \\
& =\frac{\sqrt{L_{\text {edge }} \cdot L_{S}}}{\pi \sigma^{2}} \int_{-|k|}^{\infty} \mathrm{d} x\left(1+\frac{x}{|k|}\right) \cdot\left(1+\frac{x-u}{|k|}\right) \\
& \quad \cdot \exp \left(-\frac{L_{\text {edge }} x^{2}}{\sigma^{2}}\right) \exp \left(-\frac{L_{S}(x-u)^{2}}{\sigma^{2}}\right) .
\end{aligned}
$$

If $|k|$ is large, the following approximation is valid:

$$
\begin{aligned}
p_{u}(u) \approx & \frac{\sqrt{L_{\text {edge }} \cdot L_{S}}}{\pi \sigma^{2}} \int_{-\infty}^{\infty} \mathrm{d} x \\
& \cdot \exp \left(-\frac{L_{\text {edge }} x^{2}}{\sigma^{2}}\right) \exp \left(-\frac{L_{S}(x-u)^{2}}{\sigma^{2}}\right),
\end{aligned}
$$

which is the pdf of the difference of two Gaussian random variables. Hence it follows that $z$ defined in Eqn. (32) is a standard Gaussian random variable. In fact, it can be easily verified experimentally that the approximation given in Eqn. (39) is quite good even for $k / \sigma \approx 0.5$.

Concluding this section, the procedure of assigning statistical significance to a watershed dam consists of the following steps:

- Calculate and average the components of the gradient along a watershed dam and then calculate the magnitude of the average.

- Calculate and average the components of the gradient within the subset $S$ of the watershed basin and then calculate the magnitude of the average.

- Calculate the statistics $z$, given in Eqn. (32), and test, assuming some significance level, whether this is significantly different from zero.

\section{Growth model}

Generally, the problem of finding a simply-connected set of pixels $S$, which contains the pixel with the lowest gradient magnitude within the watershed basin and such that the gradient magnitudes within $S$ can be approximated by a plane of a constant gray level, can be reformulated as a properly defined growth process. The growth starts from an initial decomposition of an analyzed image into flat zones, as from the definition of flat zones they are planes of constant gray levels. Then the flat zone corresponding to the pixel with the lowest gradient magnitude within a basin is eventually merged with groups of neighboring flat zones. The merge step is accepted only if the gray levels within the set of merged flat zones can be approximated with a plane of a constant gray level. This condition can be tested with appropriate statistical tools. Here, the multiple regression model is used and the plane is considered to be of a constant gray level if the corresponding coefficients of the regression model are not significantly different from zero, given some significance level.

To the best of the author's knowledge, the computational complexity of such a procedure is exponential. Thus in the present study the following growth process was performed to find $S$ : an empty stack is created for every gray level in the image. The growth starts from 

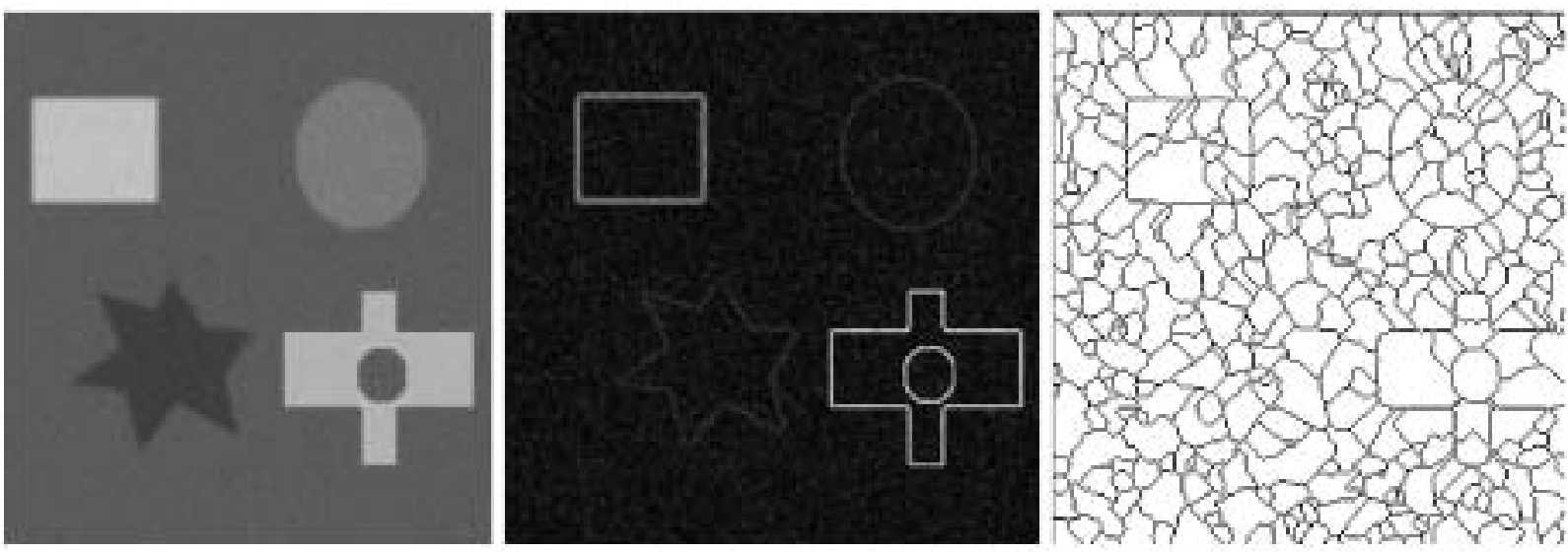

(a)

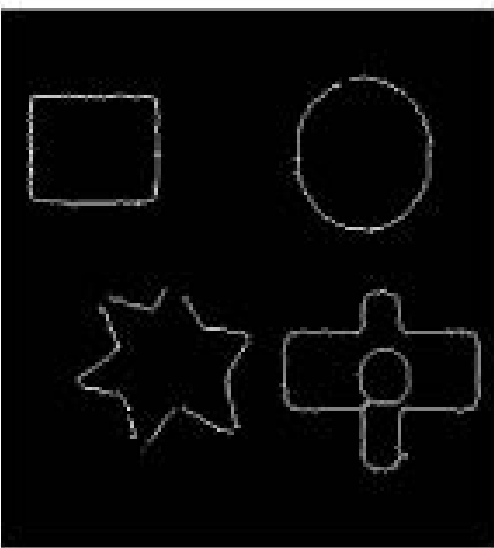

(d)

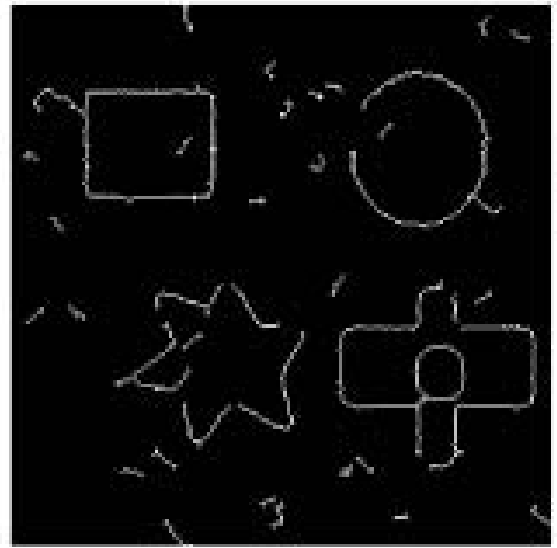

(e)

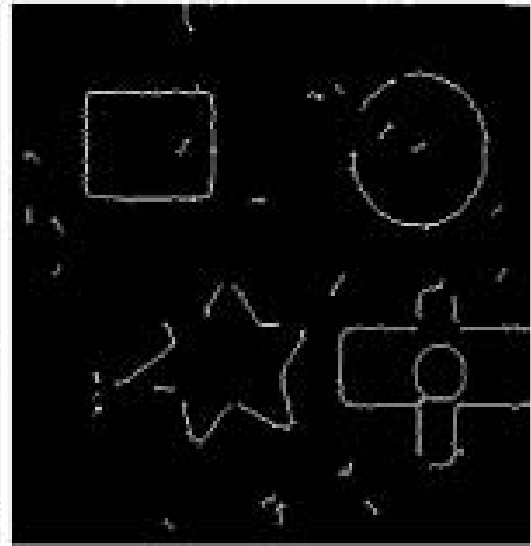

(f)

Fig. 9. (a) Artificially generated noisy image, (b) its magnitude gradient image calculated using a two-point operator, (c) watersheds of (b), (d) watersheds significant at the $10^{-5}$ significance level, (e) watershed dams with morphological dynamics higher than three, (f) watershed dams with morphological dynamics higher than four.

the pixel with the minimal gradient amplitude within the basin. The neighbors of the initial point are added to the heaps corresponding to the gray levels of these pixels. Next, the growth starts from the pixel at the top of the non-empty stack having the lowest gray-level. This pixel is removed from the stack and its neighbors are added to corresponding stacks. Only pixels belonging to the same basin as the initial pixels are considered in the growth. The growth is terminated if all stacks become empty or if the size of $S$ exceeds some limiting value. Every $m$ iterations ( $m=5$ was used in this study), it is checked if the gray-levels within the cluster of pixels can be approximated with a plane of a constant gray level. If this condition is fulfilled, the configuration of pixels is stored.

\section{Results and discussion}

The drawbacks of morphological dynamics manifest themselves in a most striking manner when analyzing images with the gray-level function equal to some constant plus Gaussian noise. While, intuitively, the dynamics of such an image should be zero, morphological dynamics are not. The distribution of gradient magnitudes is given by Eqn. (15) in such a case. Assuming that the mean area of a basin is equal to $L$ pixels ( $L$ is roughly equal to the inverse of the probability of obtaining such $3 \times 3$ configuration of pixels that a minimal gray-level is in the center 


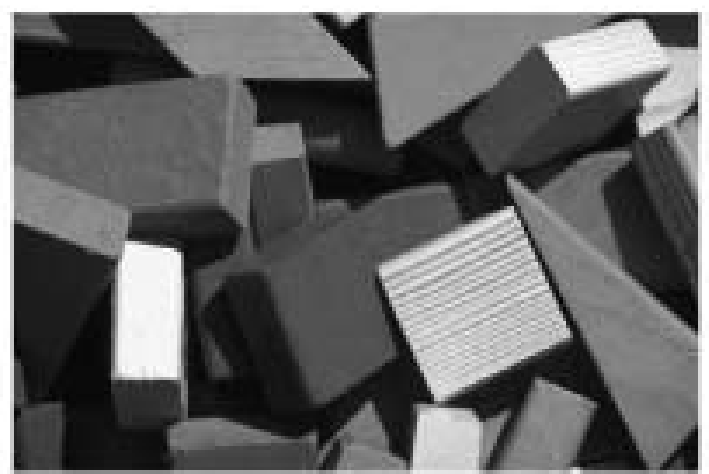

(a)

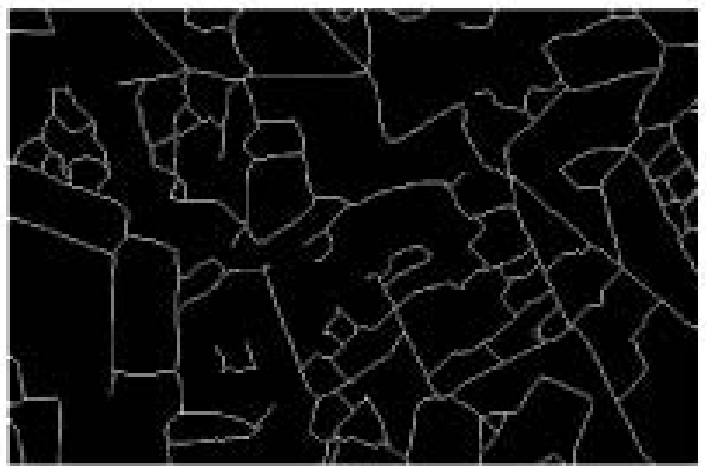

(c)

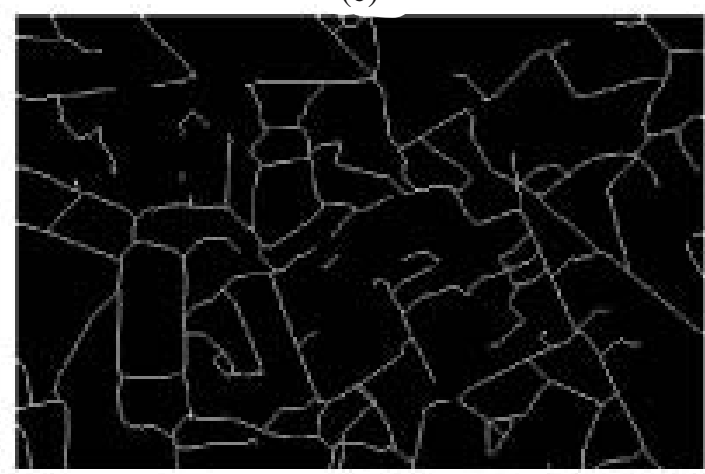

(e)

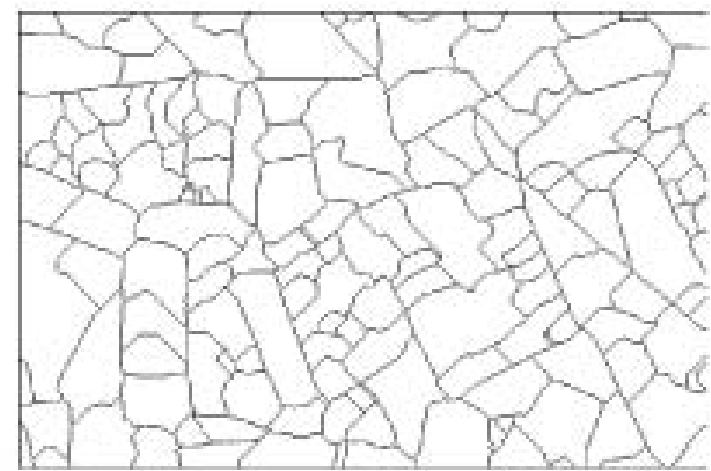

(b)

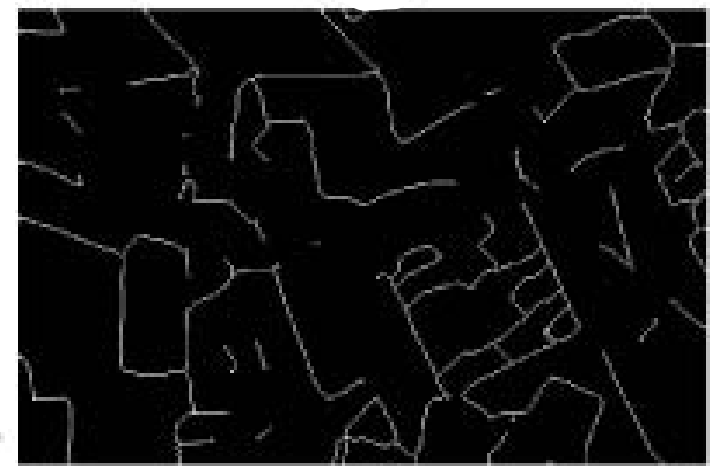

(d)

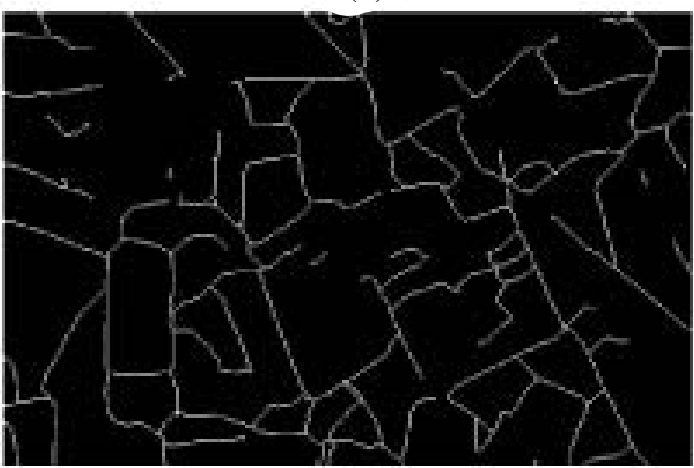

(f)

Fig. 10. (a) Image of a real scene (blocks), (b) watersheds of the gradient magnitude of (a), (c) watershed dams with morphological dynamics higher than 0 , (d) watershed dams with morphological dynamics higher than one, (e) watersheds significant at the 0.06 significance level, (f) watersheds significant at the 0.02 significance level.

of a $3 \times 3$ cluster. If a Gaussian filter with the kernel of size $s$ is applied to a noise image, then $L$ is proportional to the square of $s$, because only minima global within the filter support survive filtering) and the length of a dam is proportional to the square root of $L$, it follows from Eqn. (20) that morphological dynamics are proportional to $L^{-1 / 4}-L^{-1 / 2}$, hence equal to zero only in the limit of an infinite $L$. In contrast, the expected value of statistical dynamics is equal to zero independently of $L$.

To further test the performance of the proposed procedure of calculating statistical dynamics, it was applied to images of artificial structures (a sample structure is pre- sented in Fig. 9). Both morphological and statistical dynamics were calculated according to Eqns. (5) and (32), respectively. In Fig. 9(d), only watershed dams significant at the $10^{-5}$ level were left $(z>4.26)$. In Figs. 9(e) and 9(f), watershed dams with morphological dynamics higher than 3 and 4, respectively, were left. Clearly, increasing the threshold for morphological dynamics results in removing both false and true edges from the watershed image. In contrast, statistical dynamics are quite robust to noise. In fact, almost all artificial dams are removed at the $10^{-3}$ significance level.

The procedure for statistical dynamics was also ap- 


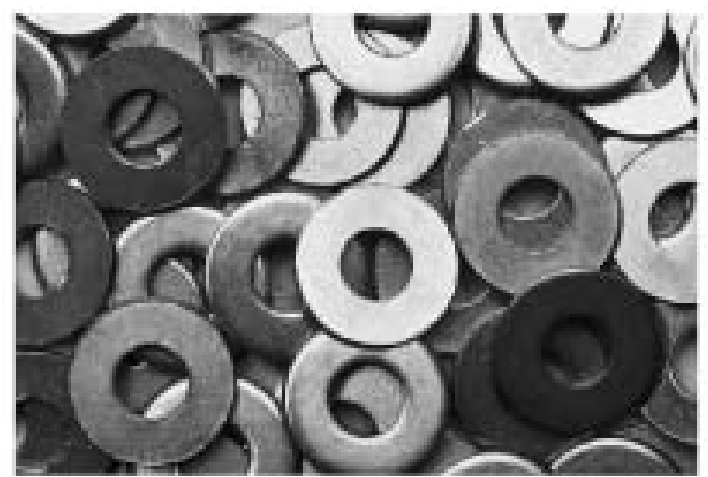

(a)

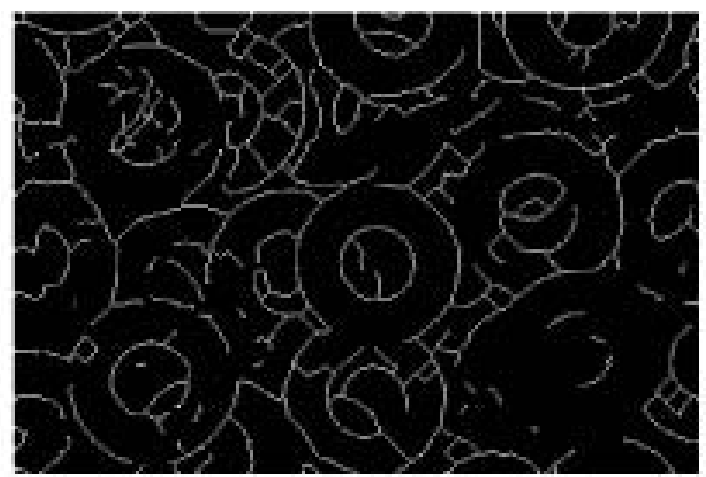

(c)

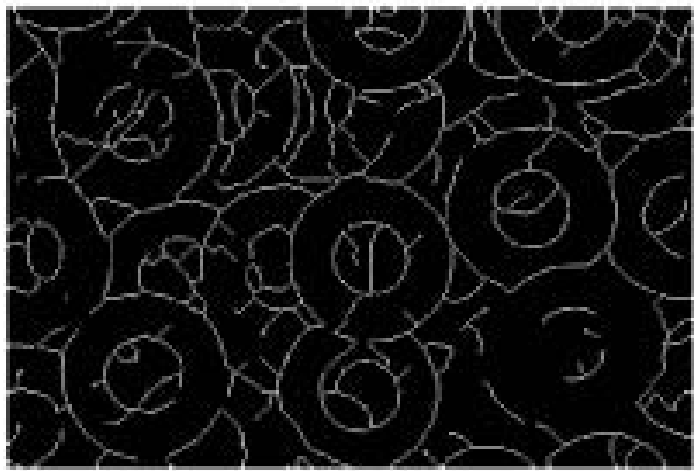

(e)

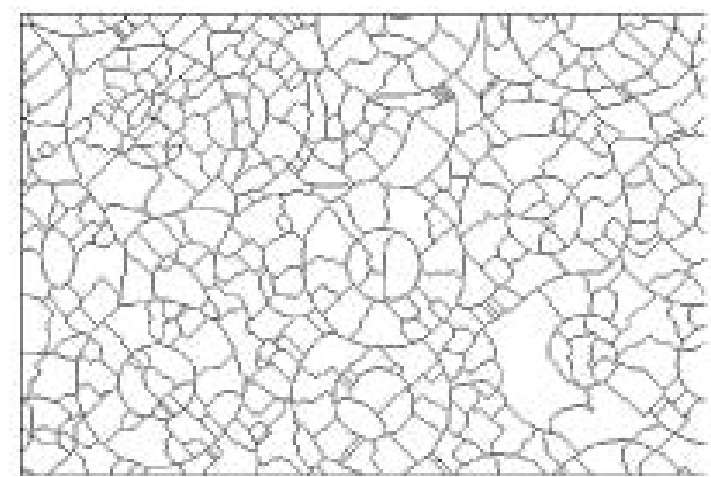

(b)

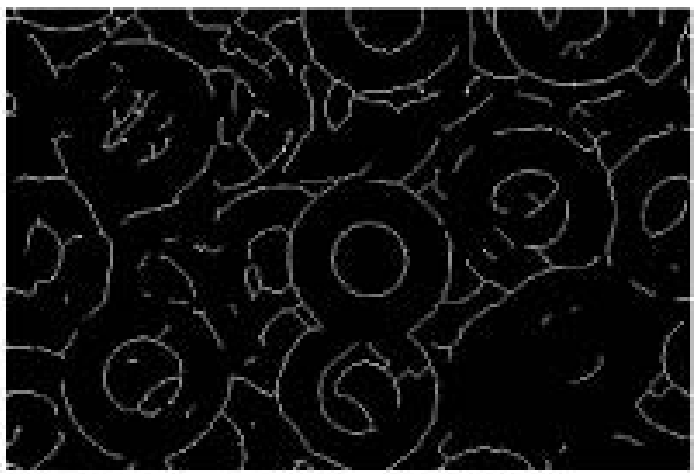

(d)

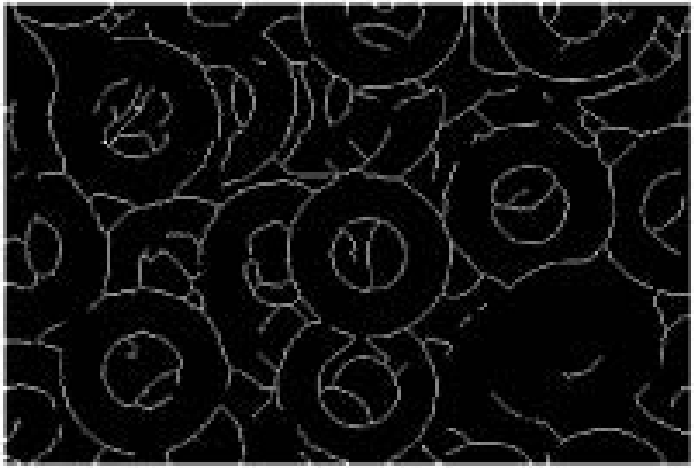

(f)

Fig. 11. (a) Image of a real scene (washers), (b) watersheds of the gradient magnitude of (a), (c) watershed dams with morphological dynamics higher than four, (d) watershed dams with morphological dynamics higher than six, (e) watersheds significant at the $2 \times 10^{-7}$ significance level, (f) watersheds significant at the $10^{-12}$ significance level.

plied to images of real scenes (Figs. 10 and 11). The actual values of the dispersion of noise are not known in that case and the significance levels in the caption were calculated assuming $\sigma=5$. In contrast, morphological dynamics are an absolute quantity. It is, however, clear from the images that even if the actual noise intensity and the noise model are not known, the evaluation of watershed dams with statistical dynamics better removes false dams than morphological dynamics.

In the derivations presented in the previous sections, the properties of a gradient image, generated with the sim- plest, two-point gradient operator, were analyzed. Clearly, if one replaces the two-point gradient operator with another linear gradient kernel, involving $n$ points instead of two, $\sigma / \sqrt{2}$ in the denominator on the right-hand side of Eqn. (32) must be replaced with $\sigma / \sqrt{n}$. If the standard deviation of noise is not known, its value can be removed from Eqn. (32) with the cost that statistical significance can be no longer assigned to the watershed dams.

The proposed definition of statistical dynamics can be extended to the case of non-uniform noise. In many important cases of image acquisition devices, the photon 
counting, underlying image acquisition, is a Poisson process with the standard deviation inversely proportional to the square root of the number of registered photons. Usually, the assumption about the Gaussian distribution of noise is reasonable because the Poisson distribution approaches the Gaussian one for a large number of registered photons. However, for linear detectors, the standard deviation of noise is inversely proportional to the square root of the gray-level intensity and may change across an image if the gray-level intensity function is not constant. This phenomenon can, however, be easily accounted for if Eqn. (32) is appropriately modified (for example, $z$ can be multiplied by a square root of the mean gray level intensity within a basin).

A growth procedure analogous to the one used for watershed basins can also be applied to watershed dams, provided that the growth is restricted only to pixels contained within the watershed and that the growth cannot pass through nodes of the graph associated with the watershed. In an extreme case, if planes of constant gray levels associated with pixels having the lowest gradient magnitudes within basins and dams cannot be found, then statistical dynamics reduce to morphological ones.

The watershed transform creates closed-curve representations of object boundaries, even if the boundary is not actually closed. Common statistical tools can be used to find gaps within watershed dams and to evaluate dams appropriately.

In conclusion, in was shown in this study that morphological dynamics provide a biased estimate of the intensity jump across an object boundary. An unbiased estimate of the difference of gradient magnitudes between a watershed dam and a neighboring watershed basin was derived. The proposed statistical definition of contour dynamics allows defining a hierarchy of partitions, based on the significance level of watershed dams.

\section{References}

Beucher, S. and Lantuéjoul, C. (1979). Use of watersheds in contour detection, Proceedings of the International Workshop on Image Processing, Real-Time Motion Detection/Estimation, Rennes, France, pp.17-21.

Beucher, S. and Meyer, F. (1993). The morphological approach to segmentation: The watershed transformation, in E. Dougherthy (Ed.), Mathematical Morphology in Image Processing, Marcel Dekker, New York, NY, pp.433-481.
Beucher, S. (1994). Watershed, hierarchical segmentation and waterfall algorithm, in J. Serra and P. Soille (Eds.), Mathematical Morphology and Its Application to Image Processing, Kluwer Academic Publishers, Dordrecht, pp.69-76.

Burry, K. V. (1975). Statistical Methods in Applied Science, John Wiley \& Sons, New York, NY.

Haris, K., Efstratiadis, S. N., Maglaveras, N. and Katsaggelos, A.K. (1998). Hybrid image segmentation using watersheds and fast region merging, IEEE Transactions on Image Processing 7(12): 1684-1699.

Haris, K., Efstratiadis, S. N. and Maglaveras, N. (2001). Hierarchical image segmentation based on contour dynamics, Proceedings of the International Conference on Image Processing, Thessaloniki, Greece, Vol. 1, pp. 54-57.

Grimaud, M. (1992). A new measure of contrast: Dynamics, Proceedings of the SPIE Conference on Image Algebra and Morphological Processing III, San Diego, CA, USA, Vol. 1769, pp.292-305.

Najman, L. and Schmitt, M. (1996). Geodesic saliency of watershed contours and hierarchical segmentation, IEEE Transactions on Pattern Analysis and Machine Intelligence 18(12): 1163-1173.

Vincent, L. and Soille, P. (1991). Watersheds in digital spaces: An efficient algorithm based on immersion simulations, IEEE Transactions on Pattern Analysis and Machine Intelligence 13(6): 583-598.

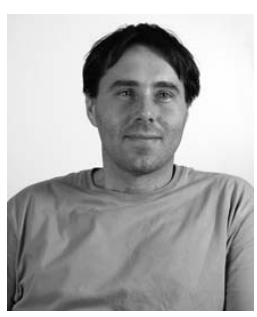

Zbisław Tabor was born in Czeladź, Poland, in 1970. He received an M.Sc. degree in physics from Jagiellonian University in Cracow, Poland, in 1994, and a Ph.D. degree from the same University in 1999. In 2000, he became an associate professor of biophysics at Jagiellonian University. Since 2006, he has been with the Cracow University of Technology, where he is an associate professor at the Image Analysis Department of the Institute of Applied Computer Science. His research interests focus on biomedical engineering, biomedical image analysis, and simulations of biological processes.

Received: 26 January 2008 Revised: 30 April 2008 